Chinese Journal of Organic Chemistry

ARTICLE

\title{
8-烯丙基山竹醇的合成及其抗癌活性研究
}

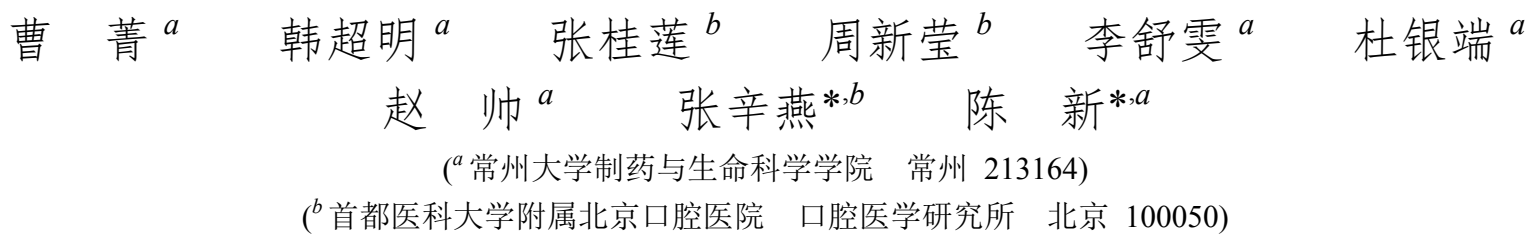

\begin{abstract}
摘要 山竹醇来源于印度藤黄, 具有抗癌和抗炎活性. 用烯丙基取代山竹醇结构中 C(8)位置上的过大支链 5-甲基-2-(1甲基乙烯基)-4-己烯基, 因为这个过大支链可能会影响山竹醇的生物活性和吸收度. 以 1,3-环己二酮为起始原料, 经过 Effenburger 环化等一系列步骤得到关键桥环中间体(土)-1-羊基-5,5-二甲基-4,6-双(3-甲基-丁-2-烯基)-二环[3.3.1]壬-2烯-3,9-二酮(8), 随后通过五步反应得到 8-烯丙基山竹醇, 并用噻唑蓝(MTT)法对其进行生物活性测试. 研究结果表明, 8-烯丙基 garcinol 对口腔鳞癌细胞具有显著的抑制增殖作用.
\end{abstract}

关键词 山竹醇; Effenburger 环化; 合成; 结构改造

\section{Synthesis and Anticancer Activity of 8-Allyl Garcinol}

\author{
Cao, Jing ${ }^{a} \quad$ Han, Chaoming ${ }^{a} \quad$ Zhang, Guilian $^{b} \quad$ Zhou, Xinying $^{b} \quad$ Li, Shuwen ${ }^{a}$ \\ Du, Yinduan $^{a} \quad Z_{\text {Zho, Shuai }}{ }^{a}$ Zhang, Xinyan ${ }^{*}, b \quad$ Chen, Xin ${ }^{*}, a$ \\ ( ${ }^{a}$ School of Pharmaceutical Engineering and Life Science, Changzhou University, Changzhou 213164) \\ ( ${ }^{b}$ Beijing Institute of Dental Research, Beijing Stomatological Hospital, Capital Medical University, Beijing 100050)
}

\begin{abstract}
Garcinol, isolated from the fruit rind of Garcinia indica, shows anti-carcinogenic and anti-inflammatory activities. However, the $\mathrm{C}(8)$ side chain of garcinol is so large that it may influence the bioactivity of the compound. 8-Allyl garcinol, in which the bulky side chain at $\mathrm{C}(8)$ position of garcinol is replaced with much smaller allyl group, was synthesized through a 12-step procedure. Starting from 1,3-cyclohexandione, the key intermediate ( \pm )-1-hydroxy-5,5-dimethyl-4,6-bis(3-methylbut2-en-1-yl)bicyclo[3.3.1]non-2-ene-3,9-dione (8) was obtained via Effenburger cyclization. Through additional 5-steps, 8-allyl garcinol was prepared. The thiazolyl blue tetrazolium bromide (MTT) results indicated that 8-allyl garcinol shows strong inhibitory activity on cell proliferation of oral cancer cell lines.
\end{abstract}

Keywords garcinol; Effenburger cyclization; synthesis; structural modification

山竹醇，英文名 Garcinol，为多聚异戊二烯基苯甲 酮 $^{[1]}$, 是从印度藤黄的干果皮中提取出来的一种黄色晶 体化合物 ${ }^{[2]}$. 在印度和东南亚地区, 印度藤黄的干果皮 用来作为一种香料, 常用于一些民间医学, 印度藤黄的 干果皮在治疗糖尿病、肥胖、溃疡和其它疾病有着重要 作用 ${ }^{[3 \sim 5]}$. 山竹醇的化学结构与含有酚羟基和 $\beta$-二酮的 姜黄素类似, 都具有很强的抗氧化活性 ${ }^{[6,7]}$. 山竹醇还具 有抗炎, 抗癌活性 ${ }^{[8 \sim 10]}$, 同时是组蛋白乙酰转化酶 p300 抑制剂 ${ }^{[11]}$. 此外, 在细胞培养研究中, 山竹醇还表现出 抗增殖作用, 诱导细胞凋亡作用, 也是环氧化酶-2 抑制

\section{剂, 5-脂氧化酶(5-LOX)抑制剂 ${ }^{[3,9,12]}$.}

我们曾报道，山竹醇通过抑制 5-LOX，对 7,12-二甲 基苯并葱(DMBA)引导的急性炎症和仓鼠口腔项囊的口 腔鳞状细胞癌有化学预防作用 ${ }^{[13]}$. 计算机建模预测, 山 竹醇与 5-LOX 的活性位点相结合从而抑制 5-LOX, 并 且, 山竹醇的 13 和 14 位的两个羟基对抑制 5-LOX 起着 至关重要的作用 ${ }^{[13]}$. 我们近期研究表明, 这两个羟基也 是山竹醇抗癌活性的必需官能团 ${ }^{[14]}$. 计算机建模还表 明, 山竹醇结构中 $\mathrm{C}(8)$ 位置上的过大支链可能会阻碍山 竹醇与 5-LOX 的活性位点相结合, 进而不能有效抑制

\footnotetext{
* Corresponding authors. E-mail: xinyanzhangzh@126.com; xinchen@cczu.edu.cn Received January 9, 2017; revised March 25, 2017; published online April 10, 2017.

Project supported by the National Natural Science Foundation of China (Nos. 21272029, 81272982).

国家自然科学基金(Nos. 21272029, 81272982)资助项目.
} 
5-LOX. 这个过大的支链还可能会导致山竹醇的抗癌活 性降低, 膜渗透性变差 ${ }^{[13]}$. 因此, 我们计划对山竹醇的 $\mathrm{C}(8)$ 位置进行结构改造, 用烯丙基代替过大的 5-甲基2-(1-甲基乙烯基)-4-已烯基支链, 合成 8-烯丙基山竹醇 (8-allyl garcinol) (图 1).

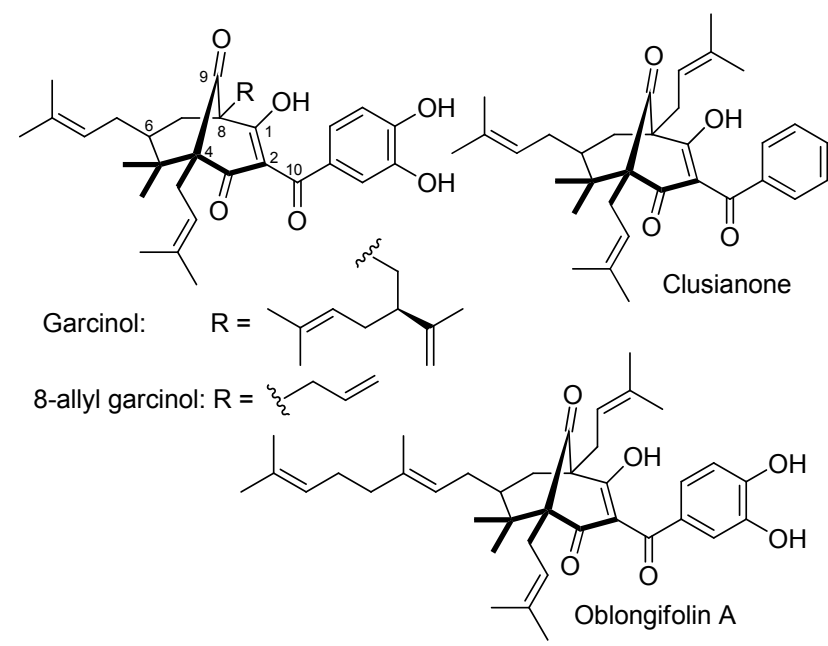

图 1 山竹醇、8-烯丙基山竹醇、Clusianone、Oblongifolin A 的化学结构

Figure 1 Chemical structure of garcinol, 8-allyl garcinol, clusianone and oblongifolin A

目前，8-烯丙基山竹醇还未见报道，合成山竹醇及 其衍生物的文献也不多. Socolsky 等 ${ }^{[15]}$ 以乙酰丙酮作为 起始原料, 经过 aldol 缩合、Tsuji-Trost 烷基化、Claisen 环化等多步反应合成得到山竹醇. Biber 等 ${ }^{[16]}$ 从乙酰丙
酩开始，通过 $\alpha$-亚甲基化、Michael 加成、Knoevenagel 缩合、烷基化、Dieckmann 缩合等反应，合成了与 8-烯 丙基山竹醇结构相似的 Oblongifolin $\mathrm{A}^{[15]}$ (图 1). Rodeschini 等 ${ }^{[17]}$ 和 Nuhant 等 ${ }^{[18]}$ 合成了与山竹醇结构相 似的 Clusianone (图 1).

\section{1 结果与讨论}

8-烯丙基山竹醇的合成路线如 Scheme 1 所示. 参考 Nuhant 等 ${ }^{[18]}$ 合成 Clusianone 的方法, 我们以 1,3-环己二 酮作为原料，以 $N, N$-二异丙基乙胺(DIEA)作为碱，与 3,3-二甲基烯丙基溴发生烷基化反应生成 3-羟基-2-(3甲基-2-丁烯基)环己-2-烯酮(2). 2 与 2-碘丙烷进行烷基 化，得到 3-异丙氧基-2-(3-甲基-2-丁烯基)环己-2-烯酮 (3), 两步产率为 $60 \%$. 在二异丙基氨基锂(LDA)作用下, 3 再与 3,3-二甲基烯丙基溴发生烷基化, 得到 3-异丙氧 基-2,6-双 (3-甲基-2-丁烯基)环己-2-烯酮(4), 产率为 $71 \%$. 化合物 4 与甲基锂反应并酸化后，以 $70 \%$ 产率生 成 3-甲基-2,4-双(3-甲基-2-丁烯基)环己-2-烯酮(5). 接 着, 5 与甲基铜锂进行 Michael 加成, 得到双甲基化产物 3,3-二甲基-2,4-双(3-甲基丁-2-烯基)环已酮(6). 经过 $\mathrm{Me}_{2} \mathrm{SO}_{4}$ 作用, 环己酮 6 被转化为甲醚化合物 7. 该中间 体无需纯化, 直接与丙二酰氯发生环化反应, 得到关键 的三环中间体 8 , 两步产率为 $27 \%$. 在对甲苯磺酸催化 下, 8 在甲醇中与原甲酸三甲酯反应，将其中的羟基保 护为甲醚 9, 以便在下一步中, 在 $\mathrm{C}(8)$ 位引入烯丙基生 成 10 .

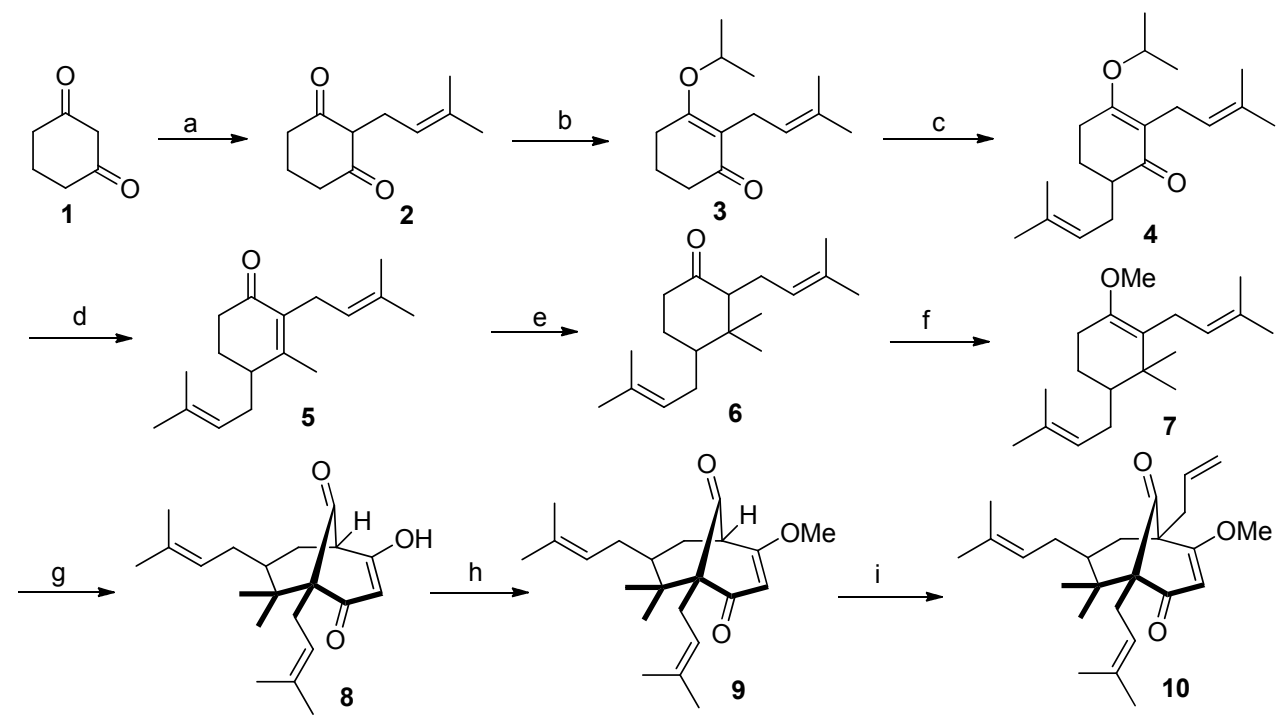

Reagents and conditions: (a) 3,3-dimethylallyl bromide, DIEA, $\mathrm{H}_{2} \mathrm{O}$, r.t., $12 \mathrm{~h}, 60 \%$; (b) i-Prl, $\mathrm{K}_{2} \mathrm{CO}_{3}, 60{ }^{\circ} \mathrm{C}, 4 \mathrm{~h}, 93 \%$; (c) LDA, 3,3-dimethylallyl bromide, $-78{ }^{\circ} \mathrm{C}, 1 \mathrm{~h}, 71 \%$; (d) MeLi, THF, $0{ }^{\circ} \mathrm{C} \sim$ r.t., $1 \mathrm{~h} ; 1 \mathrm{~mol} / \mathrm{L} \mathrm{HCl}$, r.t., $30 \mathrm{~min}, 70 \%$; (e) $\mathrm{CuBr}^{\circ} \mathrm{Me}_{2} \mathrm{~S}, \mathrm{MeMgBr}, \mathrm{HMPA}, \mathrm{TMSCl}$, THF, $-78{ }^{\circ} \mathrm{C}, 2 \mathrm{~h}, 86 \%$; (f) $\mathrm{Me}_{2} \mathrm{SO}_{4}, t$-BuOK, DMSO, r.t., $1 \mathrm{~h}$; (g) malonyl dichloride, benzyltriethylammonium chloride, ether, $-20{ }^{\circ} \mathrm{C}, 24 \mathrm{~h}$; (h) trimethyl orthoformate, TsOH, MeOH, $50{ }^{\circ} \mathrm{C}, 16 \mathrm{~h}, 81 \%$; (i) allyl bromide, LDA, THF, $-78{ }^{\circ} \mathrm{C}, 1 \mathrm{~h}, 72 \%$.

图式 1 三环中间体 10 的合成

Scheme 1 Synthetic route for tricyclic intermediate 10 
Rodeschini 等 ${ }^{[17]}$ 利用三环化合物 1-甲氧基-5,5-二甲 基-4,6,8-三(3-甲基-丁-2-烯基)二环[3.3.1]壬-2-烯-3,9-二 酮(A1)与苯甲酰氯在强碱 2,2,6,6-四甲基哌啶锂(LTMP) 作用下进行酰基化反应, 所得产物 2-苯甲酰基-1-甲氧 基-5,5-二甲基-4,6,8-三(3-甲基-丁-2-烯基)二环[3.3.1]壬2-烯-3,9-二酮(A2)再经过 $\mathrm{LiOH}$ 水解而得到 Clusianone (Scheme 2). 然而, 在此条件下, 我们尝试了 10 与 3,4二甲氧基苯甲酰氯反应, 却无法得到预期的酰基化产物 11. 我们将 10 在 $\mathrm{LiOH}$ 作用下进行水解, 以 $60 \%$ 产率得 到 12 (Scheme 3). 此反应看似简单, 却易导致很低的产 率, 关键是要控制好反应温度和反应时间, 因为 $\mathbf{1 0}$ 不太 容易被水解, 但温度过高或者反应时间过长, 却可能使 三环中间体分解. 用 3,4-二甲氧基苯甲酰氰 ${ }^{[18]}$ 取代 3,4二甲氧基苯甲酰氯后, 在三环化合物 $12 \mathrm{C} 2$ 位上可以顺 利进行酰化. 对于 3,4-二甲氧基苯甲酰氰和相应酰氯同
三环中间体(10 和 12)亲电反应的差异, 我们推测其可能 原因是 “氧基效应” [19]，也就是酰氰化合物的氰基能 与烯醇化合物形成氢键, 而且在有机溶剂中呈现强碱性 质，因此，酰氰比酰氯更有利于亲电取代反应.

不过，所得产物 13 在进行去甲基化反应时，并未得 到目标产物(Scheme 3). 可能的原因是, 13 在低温(一 $\left.78{ }^{\circ} \mathrm{C}\right)$ 下反应不完全，单羟基和双羟基化产物混在一 起，不易有效分离; 但若提高反应温度，三溴化硼则会 破坏三环骨架.

最终, 我们改变了酚羟基的保护基, 用 3,4-二乙酰 氧基苯甲酰㲵代替 3,4-二甲氧基苯甲酰氰，再与化合物 12 进行酰基化反应，以 $73 \%$ 产率得到了酰基化物 14. 最 后，14 在碳酸钾和甲醇体系中进行水解，以 $64 \%$ 产率顺 利得到目标产物 8-烯丙基山竹醇(Scheme 4).

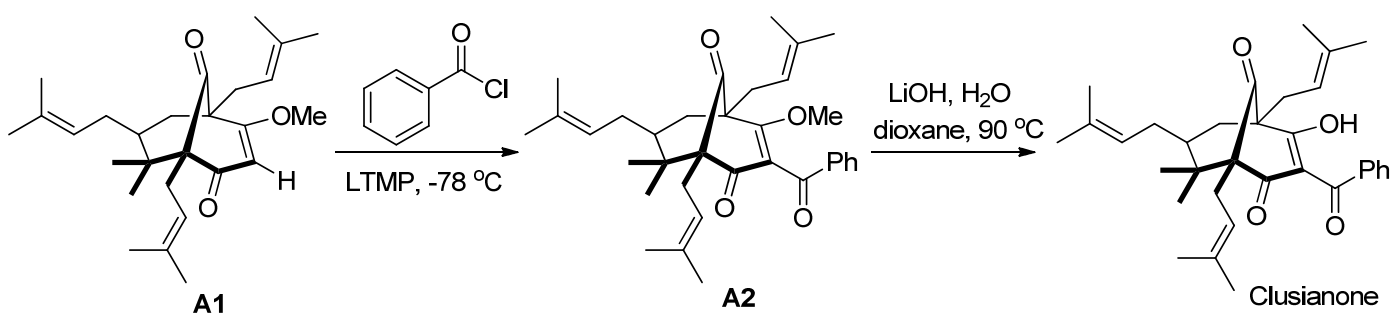<smiles>C=CCC12C(=O)C=C(C)C(CC=C(C)C)(C(=O)C=C1CC=C(C)C)C2(C)C</smiles><smiles>COc1ccc(C(=O)Cl)cc1OCC(C)(C)C(=O)O</smiles><smiles>C=CC12CC(CC=C(C)C)C(CC=C(C)C)(C(=O)C1=O)C(C)(C)C2C(=O)c1ccc(OC)c(OC)c1</smiles>

图 2 三环中间体与苯甲酰氯的酰基化反应

Scheme 2 Acylation of the tricyclic intermediates with benzoyl chlorides

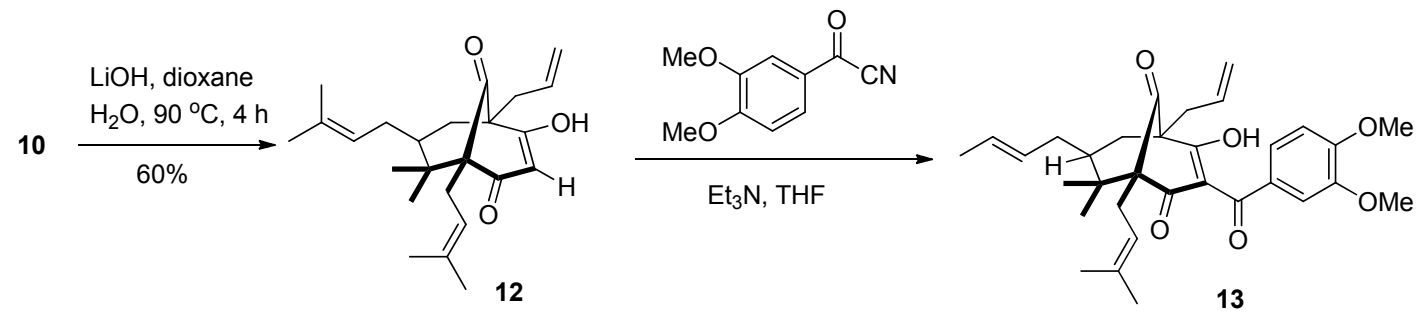

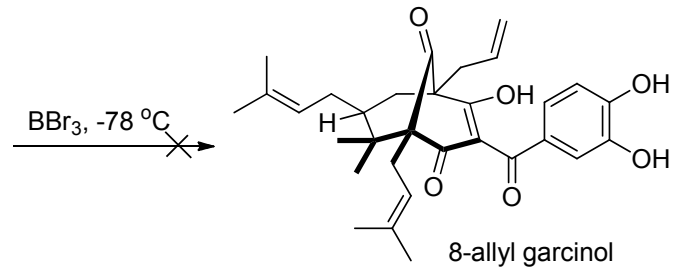

图式 3 三环中间体与苯甲酰氰的酰基化反应

Scheme 3 Acylation of the tricyclic intermediates with benzoyl cyanides 


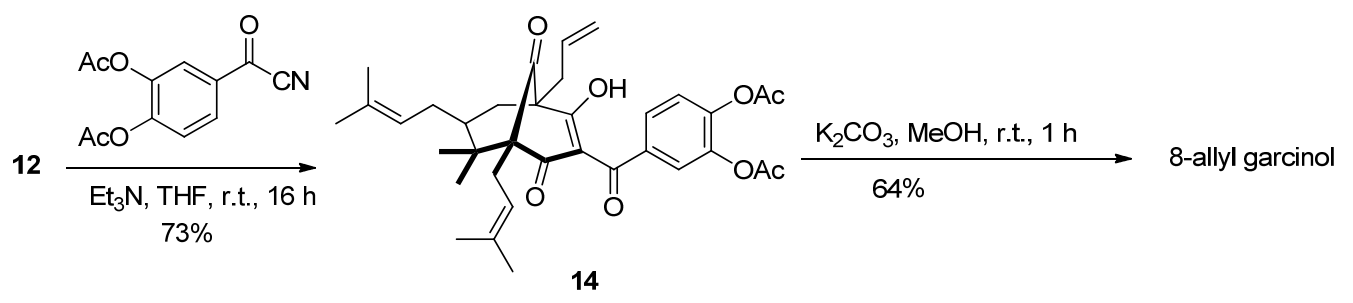

图式 4 8-烯丙基山竹醇的合成

Scheme 4 Synthesis of 8-allyl garcinol

\section{2 山竹醇和 8-烯丙基山竹醇的生物活性比较}

我们利用噻唑蓝(MTT)法, 比较了山竹醇和 8-烯丙 基山竹醇对口腔鳞癌细胞 SCC-15 和 CAL-27 细胞增殖 的抑制活性. 与阴性对照组相比, 山竹醇和 8-烯丙基山 竹醇均能够显著抑制口腔鳞癌细胞 SCC-15 和 CAL-27 细胞的增殖, 差异具有统计学意义 $(P<0.01)$. 随着山竹 醇和 8-烯丙基山竹醇浓度的增高和作用时间延长, 对 SCC-15 和 CAL-27 细胞的抑制作用逐渐增强, 呈一定浓 度和时间依赖关系(图 1 和图 2). 本研究发现, 在药物低 至中浓度 $(5 \sim 20 \mu \mathrm{mol} / \mathrm{L})$ 时, 8 -烯丙基山竹醇的抑制效果 优于山竹醇 $(P<0.01)$.

\section{2 结论}

以 1,3 -环已二酮为起始原料, 采用了 Effenburger 环化为关键步骤, 经过 12 步反应得到了 8-烯丙基山竹 醇, 总产率为 $1.2 \%$. 该方法避免了使用贵重试剂和有毒 试剂, 操作简单, 为山竹醇新衍生物的合成提供了参考, 具有潜在的应用价值. 并且, 利用 MTT 法测得 8-烯丙 基山竹醇对人口腔鳞癌细胞具有显著的抑制增殖作用.

\section{3 实验部分}

\section{1 仪器与试剂}

熔点使用上海申光公司的显微熔点仪, 型号为 SGW X-4B; NMR 用 Bruker (300 和 $400 \mathrm{MHz}$ ) 型核磁共 振仪测定 ( $\mathrm{CDCl}_{3}, \mathrm{DMSO}-d_{6}$ 作溶剂, TMS 为内标); HRMS 用 Thermo Orbitrap Elite 测定; 昆山市超声仪器 有限公司 SQ-5200 型超声波清洗器; 160 200 目硅胶 (青岛海洋化工厂生产); 薄层色谱使 HSGF-254(购自烟 台江友硅胶开发有限公司). 试剂均为分析纯.

\section{2 实验方法}

\subsubsection{3-羟基-2-(3-甲基-2-丁烯基)环已-2-烯酮(2)的合} 成

取 1,3-环已二酮 $(50 \mathrm{~g}, 446.2 \mathrm{mmol})$ 溶于水中 $(100$ $\mathrm{mL}$ ), 冷却至 $0{ }^{\circ} \mathrm{C}$, 向其中缓慢加入 $N, N$-二异丙基乙胺 (57.53 g, $446.2 \mathrm{mmol}$ ), 之后再缓慢加入 3,3-二甲基烯丙 基溴(73.08 g, $491 \mathrm{mmol}$ ), 在室温搅拌过夜(TLC 监测反
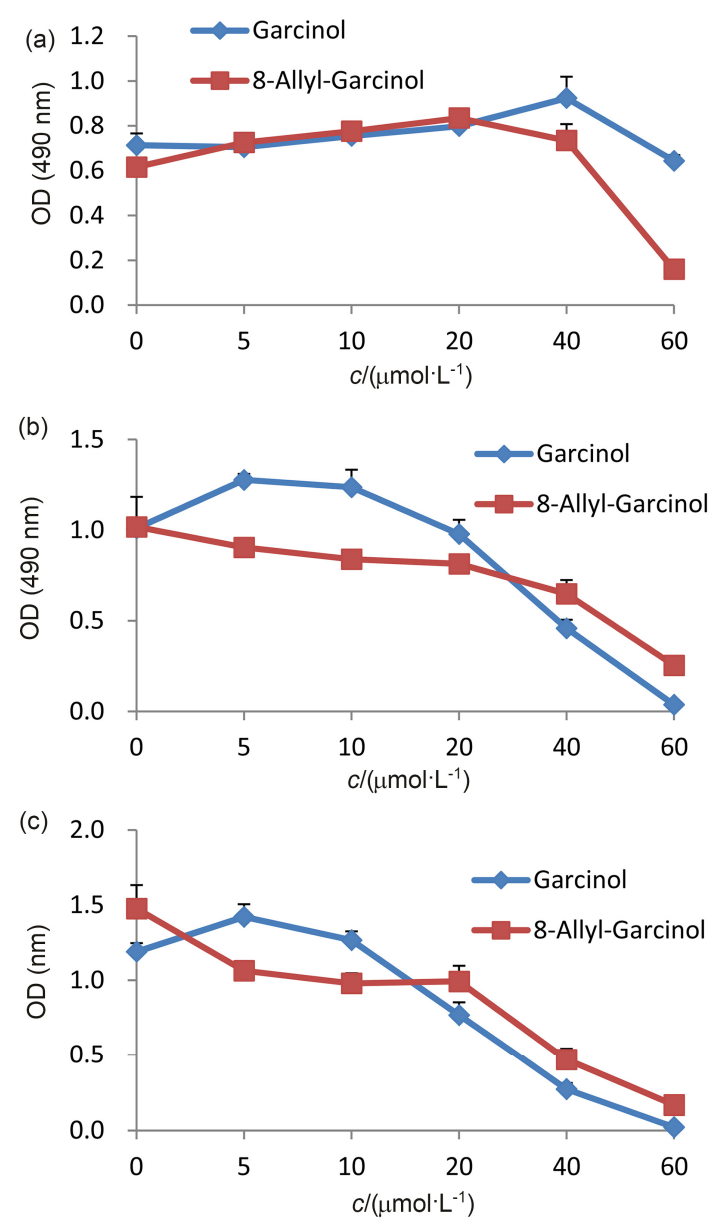

图 1 不同浓度 gacinol 和 8-烯丙基 garcinol 处理 CAL-27 细胞 24 (a)、 48 (b) 和 72 (c) h 的 OD 值

Fig. 1 OD values of CAL-27 cells treated with different concentrations of garcinol and 8-allyl garcinol at 24 (a), 48 (b) and 72 (c) h

应进程). 反应毕，向反应液加入 $2 \mathrm{~mol} / \mathrm{L} \mathrm{NaOH}$ 溶液, 调节 $\mathrm{pH} \approx 11$, 分液, 水相用乙酸乙酯萃取 $(300 \mathrm{~mL})$, 之 后用冰醋酸酸化，直到白色固体不再析出，此时 $\mathrm{pH} \approx 3$. 抽滤，并放在真空干燥箱干燥，得到白色固体 2 (48.16 g, 产率 60\%). m.p. 141 $145{ }^{\circ} \mathrm{C} ;{ }^{1} \mathrm{H}$ NMR $(400 \mathrm{MHz}$, DMSO- $\left.d_{6}\right) \delta: 5.00(\mathrm{t}, J=7.1 \mathrm{~Hz}, 1 \mathrm{H}), 2.81(\mathrm{~d}, J=7.0 \mathrm{~Hz}$, $2 \mathrm{H}), 2.31 \sim 2.28(\mathrm{~m}, 4 \mathrm{H}), 1.85 \sim 1.78(\mathrm{~m}, 2 \mathrm{H}), 1.63(\mathrm{~s}$, $3 \mathrm{H}), 1.59(\mathrm{~s}, 3 \mathrm{H}) ;{ }^{13} \mathrm{C}$ NMR (100 MHz, DMSO- $\left.d_{6}\right) \delta$ : 

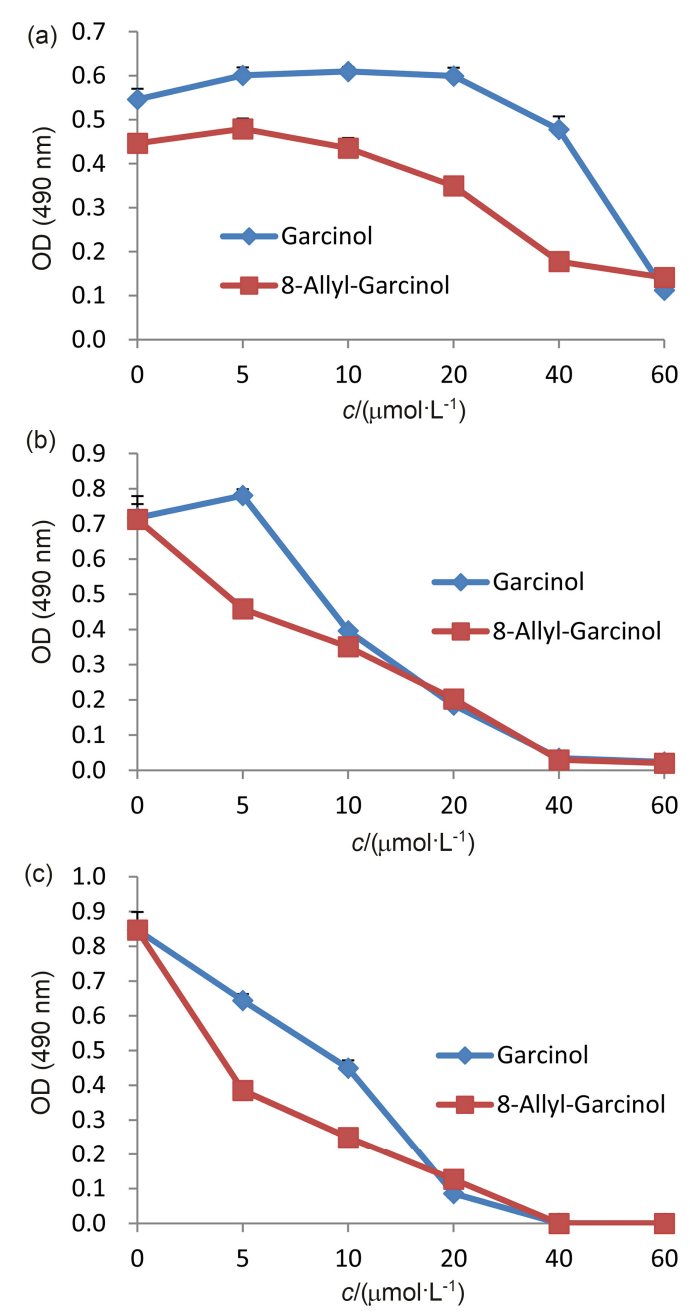

图 2 不同浓度 gacinol 和 8-烯丙基 garcinol 处理 SCC-15 细胞 24 (a)、 48 (b) 和 72 (c) $h$ 的 OD 值

Figure 2 OD values of SCC-15 cells treated with different concentrations of garcinol and 8-allyl garcinol at 24 (a), 48 (b) and $72(\mathrm{c}) \mathrm{h}$

129.74, 124.23, 114.70, 33.01, 26.06, 21.25, 21.13, 18.18; HRMS calcd for $\mathrm{C}_{11} \mathrm{H}_{17} \mathrm{O}_{2}[\mathrm{M}+\mathrm{H}]^{+}$181.1223; found $181.1229 .$.

3.2 .23 -异丙氧基-2-(3-甲基-2-丁烯基)环已-2-烯酮(3) 的合成

取化合物 2 (35.27 g, $196 \mathrm{mmol})$ 溶于丙酮 $(300 \mathrm{~mL})$ 中, 加入异丙基碘(116.62 $\mathrm{g}, 686 \mathrm{mmol}$ )和碳酸钾(64.04 $\mathrm{g}, 784 \mathrm{mmol}$ ), 在 $60{ }^{\circ} \mathrm{C}$ 下反应 $4 \mathrm{~h}, \mathrm{TLC}$ 检测. 反应结束 后温度降至室温, 浓缩得到粗产物, 向粗产物中加入水 $(400 \mathrm{~mL})$ 和二氯甲烷 $(400 \mathrm{~mL})$ 溶解粗产物. 溶液分液, 水相用二氯甲烷萃取 $(300 \mathrm{~mL} \times 3)$, 合并有机相, 有机相 用饱和碳酸钠溶液洗 $(200 \mathrm{~mL})$, 水洗 $(200 \mathrm{~mL})$, 饱和食 盐水洗 $(200 \mathrm{~mL})$, 无水硫酸钠干燥, 浓缩, 并进行柱层 析纯化 [洗脱剂: $V$ (石油醚) : $V$ (乙酸乙酯 $)=10: 1$ ], 得 到黄色液体 3 (40.4 g, 产率 93\%). ${ }^{1} \mathrm{H}$ NMR $(300 \mathrm{MHz}$,
$\left.\mathrm{CDCl}_{3}\right) \delta: 5.06 \sim 5.02(\mathrm{~m}, 1 \mathrm{H}), 4.57 \sim 4.51(\mathrm{~m}, 1 \mathrm{H}), 2.94$ (d, $J=7.2 \mathrm{~Hz}, 2 \mathrm{H}), 2.50(\mathrm{t}, J=6.2 \mathrm{~Hz}, 2 \mathrm{H}), 2.33(\mathrm{t}, J=$ $6.2,2 \mathrm{H}), 2.03 \sim 1.91(\mathrm{~m}, 2 \mathrm{H}), 1.69(\mathrm{~s}, 3 \mathrm{H}), 1.62(\mathrm{~s}, 3 \mathrm{H})$, $1.28(\mathrm{~s}, 3 \mathrm{H}), 1.26(\mathrm{~s}, 3 \mathrm{H}) ;{ }^{13} \mathrm{C} \mathrm{NMR}\left(75 \mathrm{MHz}, \mathrm{CDCl}_{3}\right) \delta$ : 197.24, 169.64, 129.46, 121.93, 119.38, 69.15, 35.63, 24.71, 24.65, 22.00, 20,19, 20.51, 16.80; HRMS calcd for $\mathrm{C}_{14} \mathrm{H}_{23} \mathrm{O}_{2}[\mathrm{M}+\mathrm{H}]^{+}$223.1693; found 223.1699.

3.2.3 3-异丙氧基-2,6-双(3-甲基-2-丁烯基)环已-2-烯酮 (4) 的合成

量取 THF (100 mL)加入烧瓶中, 在氮气保护下, 加 入二异丙胺 $(10.39 \mathrm{~g}, 103 \mathrm{mmol})$, 温度降至 $-78{ }^{\circ} \mathrm{C}$, 之 后缓慢滴加正丁基锂 $\left(2.5 \mathrm{~mol} / \mathrm{L}\right.$ in $\mathrm{Et}_{2} \mathrm{O}, 41.1 \mathrm{~mL}, 103$ $\mathrm{mmol}$ ), 摚拌 $30 \mathrm{~min}$, 再缓慢加入溶于 THF 化合物 $\mathbf{3}$ (20.74 g, $93 \mathrm{mmol}$ ). 在 $-78{ }^{\circ} \mathrm{C}$ 下搅拌 $1 \mathrm{~h}$ 后缓慢加入 3,3-二甲基烯丙基溴 $(16.7 \mathrm{~g}, 112 \mathrm{mmol}$ ), 在此温度下继 续摚拌 $1 \mathrm{~h}$ 后缓慢升至室温，摚拌过夜, TLC 监测. 反应 结束后，用饱和氯化铵溶液淬灭反应，分液，减压浓缩 有机相，水相用乙酸乙酯萃取 $(300 \mathrm{~mL} \times 3)$, 合并有机 相, 有机相用水洗, 饱和食盐水洗, 无水硫酸钠干燥. 减压浓缩, 粗产品通过柱层析纯化 [洗脱剂: $V($ 石油 醚) $: V($ 乙酸乙酯 $)=50 ： 1]$ 得到黄色液体 $4(18.9 \mathrm{~g}$, 产 率 71\%). ${ }^{1} \mathrm{H}$ NMR $\left(300 \mathrm{MHz}, \mathrm{CDCl}_{3}\right) \delta: 5.10 \sim 5.04(\mathrm{~m}$, $1 \mathrm{H}), 5.06 \sim 5.02(\mathrm{~m}, 1 \mathrm{H}), 4.52 \sim 4.48(\mathrm{~m}, 1 \mathrm{H}), 2.95(\mathrm{~d}, J=$ $4.3 \mathrm{~Hz}, 2 \mathrm{H}), 2.60 \sim 2.54(\mathrm{~m}, 2 \mathrm{H}), 2.48 \sim 2.40(\mathrm{~m}, 1 \mathrm{H})$, $2.18 \sim 1.12(\mathrm{~m}, 1 \mathrm{H}), 2.08 \sim 2.00(\mathrm{~m}, 2 \mathrm{H}), 1.70(\mathrm{~s}, 3 \mathrm{H})$, $1.69(\mathrm{~s}, 3 \mathrm{H}), 1.63(\mathrm{~s}, 3 \mathrm{H}), 1.60(\mathrm{~s}, 3 \mathrm{H}) ;{ }^{13} \mathrm{C} \mathrm{NMR}(75$ $\left.\mathrm{MHz}, \mathrm{CDCl}_{3}\right) \delta: 199.78,169.66,133.09,130.43,122.41$, $122.16,119.91,45.33,28.34,25.87,25.75,25.67,24.77$, 24.50, 21.89, 17.72; HRMS calcd for $\mathrm{C}_{19} \mathrm{H}_{31} \mathrm{O}_{2}[\mathrm{M}+\mathrm{H}]^{+}$ 291.2319, found 291.2321.

3.2.43-甲基-2,4-双(3-甲基-2-丁烯基)环已-2-烯酮(5) 的合成

取化合物 4 (31 g, $106.8 \mathrm{mmol})$ 加入到烧瓶中, 在氮 气保护下加入无水 $\mathrm{THF}(250 \mathrm{~mL})$, 在 $0{ }^{\circ} \mathrm{C}$ 缓满滴加甲 基锂(1.3 mol/ $/$ in $\mathrm{Et}_{2} \mathrm{O}, 124 \mathrm{~mL}, 160.2 \mathrm{mmol}$ )后，直接在 室温中反应 $30 \mathrm{~min}$, 随后再次将反应液冷却至 $0{ }^{\circ} \mathrm{C}$ 并缓 慢滴加稀盐酸, 调节至弱酸性并搅拌 $30 \mathrm{~min}$, TLC 监测. 反应毕，分液，减压浓缩有机相，水相用乙酸乙酯萃取 $(200 \mathrm{~mL} \times 3)$, 合并有机相, 有机相用水洗, 饱和食盐水 洗, 无水硫酸钠干燥, 减压浓缩并柱层析纯化[洗脱剂: $V($ 石油醚 $): V($ 乙酸乙酯 $)=150 ： 1$ ], 得到黄色液体 $\mathbf{5}$ (23.52 g, 产率 71\%). ${ }^{1} \mathrm{H}$ NMR (400 $\left.\mathrm{MHz}, \mathrm{CDCl}_{3}\right) \delta$ : $5.11 \sim 5.06(\mathrm{~m}, 1 \mathrm{H}), 4.89 \sim 4.82(\mathrm{~m}, 1 \mathrm{H}), 3.03 \sim 2.92(\mathrm{~m}$, 2H), 2.41 (ddd, $J=17.3,12.2,5.2 \mathrm{~Hz}, 1 \mathrm{H}), 2.29$ (dt, $J=$ 17.2, 4.9 Hz, 1H), 2.25 2.09 (m, 2H), 2.14 2.11 (m, 
$1 \mathrm{H}), 1.98 \sim 1.90(\mathrm{~m}, 1 \mathrm{H}), 1.94(\mathrm{~s}, 3 \mathrm{H}), 1.85 \sim 1.80(\mathrm{~m}$, $1 \mathrm{H}), 1.83$ (s, 3H), 1.82 (s, 3H), 1.81 (s, 3H), 1.80 (s, 3H); ${ }^{13} \mathrm{C}$ NMR $\left(100 \mathrm{MHz}, \mathrm{CDCl}_{3}\right) \delta: 198.91,159.53,135.64$, $134.27,132.09,123.08,122.83,42.35,34.45,30.40,26.50$, $26.37,26.21,25.15,20.68,18.51$; HRMS calcd for $\mathrm{C}_{17} \mathrm{H}_{27} \mathrm{O}[\mathrm{M}+\mathrm{H}]^{+}$247.2056; found 247.2061.

\subsubsection{3,3-二甲基-2,4-双(3-甲基丁-2-烯基)环已酮(6)的} 合成

取溴化亚铜二甲硫醚 $(0.512 \mathrm{~g}, 2.5 \mathrm{mmol})$ 于反应瓶 中, 在氮气保护下加入无水 THF $(250 \mathrm{~mL}),-78{ }^{\circ} \mathrm{C}$ 滴 加甲基溴化镁 ( $3 \mathrm{~mol} / \mathrm{L}$ in $\mathrm{Et}_{2} \mathrm{O}, 250 \mathrm{mmol}$ )和六甲基磷酰 三胺 $(17.92 \mathrm{~g}, 100 \mathrm{mmol})$, 搅拌 $20 \mathrm{~min}$ 后, 通过注射器滴 加溶于 THF 的 $5(12.26 \mathrm{~g}, 50 \mathrm{mmol})$ 和三甲基氯硅烷 $(10.86 \mathrm{~g}, 100 \mathrm{mmol}),-78{ }^{\circ} \mathrm{C}$ 下搅拌 $2 \mathrm{~h}$ 后, 滴加稀盐酸 至酸性, 搅拌 $30 \mathrm{~min}$, 升至室温搅拌 $10 \mathrm{~min}$, TLC 监测. 反应毕分液, 减压浓缩有机相, 水相用乙酸乙酯萃取 $(300 \mathrm{~mL} \times 3)$, 合并有机相, 有机相用水洗, 饱和食盐水 洗, 无水硫酸钠干燥. 减压浓缩, 粗产物通过柱层析分 离[洗脱剂: $V$ (石油醚) $: V($ 乙酸乙酯 $)=400: 1$ ], 得到黄 色液体 6 (11.35 g, 产率 86\%). ${ }^{1} \mathrm{H}$ NMR $(300 \mathrm{MHz}$, $\left.\mathrm{CDCl}_{3}\right) \delta: 5.05 \sim 5.01(\mathrm{~m}, 1 \mathrm{H}), 4.95 \sim 4.90(\mathrm{~m}, 1 \mathrm{H})$, $2.36 \sim 2.32(\mathrm{~m}, 1 \mathrm{H}), 2.26 \sim 2.20(\mathrm{~m}, 2 \mathrm{H}), 2.23 \sim 2.09(\mathrm{~m}$, $1 \mathrm{H}), 2.17(\mathrm{dd}, J=9.5,2.0 \mathrm{~Hz}, 1 \mathrm{H}), 2.08 \sim 1.99(\mathrm{~m}, 2 \mathrm{H})$, $1.71(\mathrm{~s}, 3 \mathrm{H}), 1.69 \sim 1.66(\mathrm{~m}, 1 \mathrm{H}), 1.63(\mathrm{~s}, 6 \mathrm{H}), 1.64(\mathrm{~s}$, $3 \mathrm{H}), 1.55 \sim 1.48(\mathrm{~m}, 1 \mathrm{H}), 1.41 \sim 1.35(\mathrm{~m}, 1 \mathrm{H}), 1.18(\mathrm{~s}$, $3 \mathrm{H}), 0.56(\mathrm{~s}, 3 \mathrm{H}) ;{ }^{13} \mathrm{C} \mathrm{NMR}\left(75 \mathrm{MHz}, \mathrm{CDCl}_{3}\right) \delta: 211.32$, $131.35,130.29,123.23,122.69,60.96,47.03,42.26,41.29$, $28.55,27.85,27.43,25.70,25.60,20.75,16.78,16.45$, 14.38; HRMS calcd for $\mathrm{C}_{18} \mathrm{H}_{31} \mathrm{O}[\mathrm{M}+\mathrm{H}]^{+}$263.2369; found 263.2368 .

\subsection{6甲醚化合物 7 的合成}

取化合物 $6(8 \mathrm{~g}, 30.5 \mathrm{mmol})$ 溶于干燥的 DMSO $(70 \mathrm{~mL})$, 并向其中加入叔丁醇钾 $(5.13 \mathrm{~g}, 45.8 \mathrm{mmol})$, 在 室温下搅拌 30min, 随后缓慢滴加 $\mathrm{Me}_{2} \mathrm{SO}_{4}(5.78 \mathrm{~g}, 45.8$ $\mathrm{mmol})$, 搅拌 $1 \mathrm{~h}$, 之后向其中加入石油醚 $(200 \mathrm{~mL})$, 分 液, DMSO 层用石油醚萃取 $(100 \mathrm{~mL} \times 2)$, 石油醚相用水 洗, 饱和食盐水洗, 无水硫酸钠干燥. 浓缩直接投下一 步.

3.2.7（土）-4-羟基-8,8-二甲基-1,7-双(3-甲基-丁-2-烯 基)-二环[3.3.1]壬-3-烯-2,9-二酮(8)的合成

取混合物 7 (5.94 g, $21.6 \mathrm{mmol})$ 溶于乙醚 $(12 \mathrm{~mL})$ 中, 在氮气保护下缓慢加入丙二酰氯 $(2.1 \mathrm{~mL}, 21.6 \mathrm{mmol})$, $-20{ }^{\circ} \mathrm{C}$ 搅拌过夜后, 再加入氢氧化钾水溶液 $(4.84 \mathrm{~g}$, $86.4 \mathrm{mmol}$ )和芐基三乙基氯化铵 (329 $\mathrm{mg}, 1.1 \mathrm{mmol}$ ), 之 后在室温下搅拌 $5 \mathrm{~h}$. 反应毕, 向反应液加入水 $(200 \mathrm{~mL})$
和石油醚 $(200 \mathrm{~mL})$, 并滴加氢氧化钠溶液调节至强碱性, 分液, 水相用石油醚萃取 $(250 \mathrm{~mL} \times 3)$, 合并有机相, 用 无水硫酸钠干燥, 减压浓缩, 得到化合物 6. 水相冰浴, 用稀盐酸酸化至强碱性, 随后用二氯甲烷萃取 (400 $\mathrm{mL} \times 3)$, 合并有机相, 无水硫酸镁干燥, 减压浓缩, 粗 产物柱层析分离纯化 [洗脱剂: $V$ (二氯甲烷) $: V$ (甲醇 $)=$ $200 ： 1$ ], 得到黄色固体 $8\left(1.94\right.$ g). m.p. $166 \sim 170{ }^{\circ} \mathrm{C} ;{ }^{1} \mathrm{H}$ NMR $\left(300 \mathrm{MHz}, \mathrm{CDCl}_{3}\right) \delta: 8.73(\mathrm{~s}, 1 \mathrm{H}, \mathrm{OH}), 5.85(\mathrm{~s}, 1 \mathrm{H})$, $4.96 \sim 4.92(\mathrm{~m}, 1 \mathrm{H}), 4.64 \sim 4.60(\mathrm{~m}, 1 \mathrm{H}), 3.71 \sim 3.68(\mathrm{~m}$, $1 \mathrm{H}), 3.15 \sim 3.11(\mathrm{~m}, 1 \mathrm{H}), 2.47 \sim 2.44(\mathrm{~m}, 2 \mathrm{H}), 2.05 \sim 2.02$ $(\mathrm{m}, 2 \mathrm{H}), 1.82 \sim 1.70(\mathrm{~m}, 3 \mathrm{H}), 1.65(\mathrm{~s}, 3 \mathrm{H}), 1.64(\mathrm{~s}, 3 \mathrm{H})$, $1.56(\mathrm{~s}, 3 \mathrm{H}), 1.55$ (s, 3H), 1.04 (s, 3H), $0.73(\mathrm{~s}, 3 \mathrm{H}) ;{ }^{13} \mathrm{C}$ NMR (75 MHz, $\mathrm{CDCl}_{3}$ ) $\delta: 207.21,191.08,183.32,132.74$, $132.25,121.43,118.81,108.34,67.01,55.15,44.73,40.04$, $31.87,27.15,24.91,24.81,22.73,22.16,17.08,16.87$, 14.98; HRMS calcd for $\mathrm{C}_{21} \mathrm{H}_{31} \mathrm{O}_{3}[\mathrm{M}+\mathrm{H}]^{+} 331.2268$, found 331.2272 .

3.2.8（土）-1-甲氧基-5,5-二甲基-4,6-二-(3-甲基-丁-2烯基)-二环[3.3.1]壬-2-烯-3,9-二酮(9)的合成

称取化合物 $8(500 \mathrm{mg}, 1.51 \mathrm{mmol})$ 加入到反应瓶中, 溶于无水甲醇 $(20 \mathrm{~mL})$ 中, 在氮气保护下加入对甲苯磺 酸 $(23 \mathrm{mg}, 0.134 \mathrm{mmol}$ )和原甲酸三甲酯 $(4.3 \mathrm{~mL}, 39$ $\mathrm{mmol})$, 在 $50{ }^{\circ} \mathrm{C}$ 回流, TLC 监测. 反应毕, 滴加两滴三 乙胺, 减压浓缩, 粗产物通过柱层析分离纯化[洗脱剂: $V$ (石油醚) $: V$ (乙酸乙酯 $)=30 ： 1]$, 得到化合物微黄固 体 9 (420 mg, 产率 81\%). m.p. $65 \sim 70{ }^{\circ} \mathrm{C} ;{ }^{1} \mathrm{H}$ NMR $\left(300 \mathrm{MHz}, \mathrm{CDCl}_{3}\right) \delta: 5.67(\mathrm{~s}, 1 \mathrm{H}), 4.87 \sim 4.85(\mathrm{~m}, 1 \mathrm{H})$, $4.62 \sim 4.57(\mathrm{~m}, 1 \mathrm{H}), 3.69(\mathrm{~s}, 3 \mathrm{H}), 3.12(\mathrm{~s}, 1 \mathrm{H}), 2.53(\mathrm{dd}$, $J=14.0,6.8 \mathrm{~Hz}, 1 \mathrm{H}), 2.35(\mathrm{dd}, J=14.0,6.5 \mathrm{~Hz}, 1 \mathrm{H})$, $2.09 \sim 2.05(\mathrm{~m}, 1 \mathrm{H}), 2.03 \sim 2.02(\mathrm{~m}, 1 \mathrm{H}), 1.97 \sim 1.96(\mathrm{~m}$, $3 \mathrm{H}), 1.94(2 \mathrm{~s}, 6 \mathrm{H}), 1.59$ (s, 3H), $1.52(\mathrm{~s}, 3 \mathrm{H}), 1.02(\mathrm{~s}, 3 \mathrm{H})$, $0.71(\mathrm{~s}, 3 \mathrm{H}) ;{ }^{13} \mathrm{C}$ NMR $\left(75 \mathrm{MHz}, \mathrm{CDCl}_{3}\right) \delta: 208.99$, $197.33,175.04,134.12,133.74,123.27,120.80,107.28$, $71.45,57.31,53.86,46.92,41.66,33.35,28.55,26.57$, 26.49, 24.71, 23.29, 18.76, 18.54, 16.65; HRMS calcd for $\mathrm{C}_{22} \mathrm{H}_{33} \mathrm{O}_{3}[\mathrm{M}+\mathrm{H}]^{+}$345.2430, found 345.2424.

3.2 .9 (土)-1-甲氧基-8-烯丙基-5,5-二甲基-4,6-二-(3甲基-丁-2-烯基)-二环[3.3.1]壬-2-烯-3,9-二酮(10)的合 成

取二异丙胺 $(0.8 \mathrm{~mL}, 5.56 \mathrm{mmol})$ 于反应瓶中, 用无 水 THF (16 mL), 在 $-78{ }^{\circ} \mathrm{C}$ 下缓慢加入正丁基锂(1.6 $\mathrm{mol} / \mathrm{L}$ in $\left.\mathrm{Et}_{2} \mathrm{O}, 3.5 \mathrm{~mL}, 5.56 \mathrm{mmol}\right)$, 加毕反应 $30 \mathrm{~min}$ 后, 再缓慢加入溶于 THF 的化合物 9 (372 mg, $1.11 \mathrm{mmol})$, 搅拌 $40 \mathrm{~min}$ 后, 加入 3 -溴丙烯 $(0.5 \mathrm{~mL}, 5.56 \mathrm{mmol})$. 反 应 $30 \mathrm{~min}$ 后用饱和氯化铵溶液淬灭反应, 分液, 减压浓 
缩有机相, 水相用乙酸乙酯萃取, 合并有机相, 有机相 用水洗, 饱和食盐水洗, 无水硫酸钠干燥, 减压浓缩, 粗产品通过柱层析分离纯化 [洗脱剂: $V$ (石油醚) : $V$ (乙 酸乙酯) $=30: 1$, 得到化合物黄色液体 $10(307 \mathrm{mg}$, 产 率 72\%). ${ }^{1} \mathrm{H}$ NMR $\left(300 \mathrm{MHz}, \mathrm{CDCl}_{3}\right) \delta: 5.82 \sim 5.68(\mathrm{~m}$, $2 \mathrm{H}), 5.07 \sim 4.90(\mathrm{~m}, 2 \mathrm{H}), 4.60(\mathrm{td}, J=5.6,1.3 \mathrm{~Hz}, 1 \mathrm{H})$, $3.71(\mathrm{~s}, 1 \mathrm{H}), 2.61 \sim 2.54(\mathrm{~m}, 2 \mathrm{H}), 2.42 \sim 2.39(\mathrm{~m}, 2 \mathrm{H})$, $2.08 \sim 2.05(\mathrm{~m}, 1 \mathrm{H}), 1.88 \sim 1.82(\mathrm{~m}, 1 \mathrm{H}), 1.68 \sim 1.65(\mathrm{~m}$, $1 \mathrm{H}), 1.65(\mathrm{~s}, 6 \mathrm{H}), 1.62 \sim 1.58(\mathrm{~m}, 1 \mathrm{H}), 1.55(\mathrm{~s}, 3 \mathrm{H}), 1.52$ (s, 3H), $1.39 \sim 1.32(\mathrm{~m}, 1 \mathrm{H}), 1.03(\mathrm{~s}, 3 \mathrm{H}), 0.70(\mathrm{~s}, 3 \mathrm{H}) ;{ }^{13} \mathrm{C}$ NMR (75 MHz, $\mathrm{CDCl}_{3}$ ) $\delta: 208.17,195.95,175.66,134.22$, 133.18, 133.07, 122.71, 120.41, 117.74, 107.64, 71.03, 56.61, 55.91, 46.16, 41.97, 39.65, 35.29, 27.91, 25.96, 25.88, 24.46, 22.77, 18.14, 17.94, 15.88; HRMS calcd for $\mathrm{C}_{25} \mathrm{H}_{37} \mathrm{O}_{3}[\mathrm{M}+\mathrm{H}]^{+}$385.2737, found 385.2740.

3.2.10 (土)-1-羟基-8-烯丙基-5,5-二甲基-4,6-二-(3甲基-丁-2-烯基)-二环[3.3.1]壬-2-烯-3,9-二酮(12)的合 成

取化合物 10 (260 mg, $0.68 \mathrm{mmol})$ 于反应瓶中, 加入 二氧六环溶解, 再加入氢氧化锂 $(142 \mathrm{mg}, 3.38 \mathrm{mmol}$ )和 水, 在氮气保护下 $90{ }^{\circ} \mathrm{C}$ 回流, 反应 $4 \mathrm{~h}$. 反应毕, 将反 应液冰浴, 滴加稀盐酸至酸性, 分液, 减压浓缩有机相, 水相用乙酸乙酯萃取, 合并有机相, 饱和食盐水洗, 无 水硫酸钠干燥, 减压浓缩, 粗产物经过柱层析分离纯化 [洗脱剂: $V$ (石油醚)：V(乙酸乙酯) $=7: 1$ ], 得到白色固 体 12 (127 mg, 60\%产率). m.p. $115 \sim 118{ }^{\circ} \mathrm{C} ;{ }^{1} \mathrm{H}$ NMR (300 MHz, DMSO- $\left.d_{6}\right) \delta: 5.71(\mathrm{~s}, 1 \mathrm{H}), 5.68 \sim 5.60(\mathrm{~m}, 1 \mathrm{H})$, $5.03 \sim 4.94(\mathrm{~m}, 3 \mathrm{H}), 4.66 \sim 4.63(\mathrm{~m}, 1 \mathrm{H}), 2.45(\mathrm{~d}, J=7.0$, $2 \mathrm{H}), 2.36(\mathrm{~d}, J=6.7,2 \mathrm{H}), 2.06 \sim 2.02(\mathrm{~m}, 1 \mathrm{H}), 1.71 \sim 1.68$ (m, 2H), $1.65(\mathrm{~s}, 3 \mathrm{H}), 1.62(\mathrm{~s}, 3 \mathrm{H}), 1.55(\mathrm{~s}, 3 \mathrm{H}), 1.53(\mathrm{~s}$, $3 \mathrm{H}), 1.35 \sim 1.25(\mathrm{~m}, 2 \mathrm{H}), 1.04(\mathrm{~s}, 3 \mathrm{H}), 0.67(\mathrm{~s}, 3 \mathrm{H}) ;{ }^{13} \mathrm{C}$ NMR (75 MHz, DMSO- $d_{6}$ ) $\delta$ : 208.30, 134.73, 132.40, $131.65,123.06,121.05,117.71,108.92,44.60,41.65$, $34.79,27.92,25.94,25.71,23.81,23.14,18.09,17.76$, 15.67; HRMS calcd for $\mathrm{C}_{24} \mathrm{H}_{33} \mathrm{O}_{3}[\mathrm{M}+\mathrm{H}]^{-} 369.2435$, found 369.2467 .

\subsubsection{1中间体 $\mathbf{1 4}$ 的合成}

取化合物 12 (77 mg, 0.21 mmol)溶于无水 THF 中, 在氮气保护下缓慢加入三乙胺 $(87 \mu \mathrm{L}, 0.63 \mathrm{mmol})$ 和溶 于 THF 的 3,4-二乙酰氧基苯甲酰氰 $(233 \mathrm{mg}, 0.95 \mathrm{mmol}$ ), 搅拌过夜. 反应毕, 加入饱和的氯化铵溶液, 分液, 减 压浓缩有机相, 水相用乙酸乙酯萃取, 合并有机相, 有 机相用水洗, 饱和食盐水洗, 无水硫酸钠干燥, 减压浓 缩, 粗产物经过柱层析分离纯化 [洗脱剂: $V$ (二氯甲 烷) $: V$ (甲醇 $)=400: 1]$, 得到淡黄色固体 $14(76 \mathrm{mg}$, 产
率 73\%). m.p. 89 91 ${ }^{\circ} \mathrm{C} ;{ }^{1} \mathrm{H}$ NMR (300 MHz, $\left.\mathrm{CDCl}_{3}\right) \delta$ : $7.52 \sim 7.41(\mathrm{~m}, 2 \mathrm{H}), 7.22 \sim 7.18(\mathrm{~m}, 1 \mathrm{H}), 5.94 \sim 5.81(\mathrm{~m}$, $1 \mathrm{H}), 5.21 \sim 5.10(\mathrm{~m}, 2 \mathrm{H}), 4.99 \sim 4.66(\mathrm{~m}, 2 \mathrm{H}), 2.78 \sim 2.65$ (m, $2 \mathrm{H}), 2.62 \sim 2.44(\mathrm{~m}, 2 \mathrm{H}), 2.35 \sim 2.32(\mathrm{~m}, 1 \mathrm{H}), 2.30(\mathrm{~s}$, $3 \mathrm{H}), 2.29(\mathrm{~s}, 3 \mathrm{H}), 2.17 \sim 1.99(\mathrm{~m}, 2 \mathrm{H}), 1.72(\mathrm{~m}, 1 \mathrm{H}), 1.69$ (s, 3H), $1.68 \sim 1.65(\mathrm{~m}, 1 \mathrm{H}), 1.61(\mathrm{~d}, J=4.4 \mathrm{~Hz}, 3 \mathrm{H})$, $1.57 \sim 1.54(\mathrm{~m}, 1 \mathrm{H}), 1.53(\mathrm{~s}, 3 \mathrm{H}), 1.40 \sim 1.37(\mathrm{~m}, 1 \mathrm{H})$, $1.24 \sim 1.22(\mathrm{~m}, 3 \mathrm{H}), 1.05 \sim 1.01(\mathrm{~m}, 1 \mathrm{H}), 0.70 \sim 0.66(\mathrm{~m}$, $3 \mathrm{H}) ;{ }^{13} \mathrm{C} \mathrm{NMR}\left(\mathrm{CDCl}_{3}, 100 \mathrm{MHz}\right) \delta: 207.15,195.54$, $192.33,167.84,167.53,145.66,141.36,135.38,134.87$, $134.64,133.53,133.21,127.94,124.85,122.75,122.07$, $120.31,118.80,115.95,71.18,59.22,48.62,47.77,42.46$, $41.67,29.70,26.08,24.84,23.75,22.62,20.77,20.61$, $18.15,17.92,16.19$; HRMS calcd for $\mathrm{C}_{35} \mathrm{H}_{43} \mathrm{O}_{8} \mathrm{Na}[\mathrm{M}+$ $\mathrm{Na}]^{+}$613.6772, found 613.6773.

\subsubsection{2(士)-8-烯丙基山竹醇的合成}

取化合物 14 (76 mg, $0.13 \mathrm{mmol})$ 用无水甲醇 $(3 \mathrm{~mL})$ 溶解后, 在氮气保护下加入碳酸钾 $(71 \mathrm{mg}, 0.51 \mathrm{mmol})$, 室温摚拌 $10 \mathrm{~min}$. 反应完全后, 加入饱和的氯化铵溶液, 用乙酸乙酯萃取三次,合并有机相, 有机相用饱和食盐 水洗, 无水硫酸钠干燥, 减压浓缩, 粗产物柱层析分离 纯化 [洗脱剂: $V$ (二氯甲烷) $: V$ (甲醇) $=80: 1$ ] , 得到产 物 $\left(23 \mathrm{mg}\right.$, 产率 64\%). ${ }^{1} \mathrm{H}$ NMR $\left(400 \mathrm{MHz}, \mathrm{CDCl}_{3}\right) \delta$ : $7.06 \sim 7.01(\mathrm{~m}, 2 \mathrm{H}), 6.61(\mathrm{~d}, J=8.2 \mathrm{~Hz}, 1 \mathrm{H}), 5.92 \sim 5.86$ (m, $1 \mathrm{H}), 5.20 \sim 5.07(\mathrm{~m}, 2 \mathrm{H}), 4.96 \sim 4.78(\mathrm{~m}, 2 \mathrm{H}), 2.73 \sim$ $2.70(\mathrm{~m}, 2 \mathrm{H}), 2.65 \sim 2.60(\mathrm{~m}, 2 \mathrm{H}), 2.11 \sim 1.99(\mathrm{~m}, 2 \mathrm{H})$, $1.70(\mathrm{~s}, 3 \mathrm{H}), 1.66(\mathrm{~s}, 3 \mathrm{H}), 1.63(\mathrm{~s}, 3 \mathrm{H}), 1.54(\mathrm{~s}, 3 \mathrm{H})$, $1.28 \sim 1.23(\mathrm{~m}, 5 \mathrm{H}), 1.13 \sim 1.10(\mathrm{~m}, 1 \mathrm{H}), 0.79 \sim 0.77(\mathrm{~m}$, $3 \mathrm{H}) ;{ }^{13} \mathrm{C} \mathrm{NMR}\left(\mathrm{CDCl}_{3}, 100 \mathrm{MHz}\right) \delta: 195.88,149.37$, $143.54,134.94,133.64,129.93,128.75,123.91,122.10$, $118.99,116.51,114.50,42.84,35.94,31.92,29.22,27.23$, 26.03, 25.29, 22.70, 18.17, 17.94, 16.29, 14.14; HRMS calcd for $\mathrm{C}_{31} \mathrm{H}_{37} \mathrm{O}_{6}[\mathrm{M}+\mathrm{H}]^{-}$505.2596, found 505.2591.

\section{2 .13 细胞增殖分析}

MTT 法检测细胞增殖: 将对数生长期的 SCC-15 和 CAL-27 细胞悬液调整浓度至 $1 \times 10^{4}$ 个 $/ \mathrm{mL}$ 后, 接种 到 96 孔培养板, 每孔 $200 \mu \mathrm{L}$, 细胞贴壁后, 根据预实验 结果, 分别加入不同浓度的 Garcinol 和 8 -烯丙基 Garcinol (5、10、20、40、60 $\mu \mathrm{mol} / \mathrm{L})$. 每个浓度设置 6 个复孔, 同时设置不加细胞的调零孔和不加药物的阴性 对照孔. 在 $37{ }^{\circ} \mathrm{C} 5 \% \mathrm{CO}_{2}$ 分别捊育 $24 、 48 、 72 \mathrm{~h}$, 每孔 加入 $20 \mu \mathrm{L}$ MTT 溶液 $(5 \mathrm{mg} / \mathrm{mL})$, 继续培养 $4 \mathrm{~h}$ 后终止培 养, 吸去孔内培养液, 每孔加入 $150 \mu \mathrm{L}$ 二甲基亚砜, 置 摇床上低速振荡 $10 \mathrm{~min}$, 使结晶物充分溶解. 在酶联免 疫检测仪波长 $\mathrm{OD} 490 \mathrm{~nm}$ 处测量各孔的吸光值. 应用 
SPSS 17.0 for windows 统计软件进行统计分析. 采用 ANOVA 检验, 以 $\alpha=0.05$ 作为检验水准.

\section{辅助材料(Supporting Information) 目标化合物的 ${ }^{1} \mathrm{H}$} $\mathrm{NMR},{ }^{13} \mathrm{C} \mathrm{NMR}$, 和 MS 数据. 这些材料可以免费从本 刊网站(http://siocjournal.cn/)上下载.

\section{References}

[1] Aggarwal, S.; Das, S. N. Tumor. Biol. 2016, 37, 7175.

[2] Saadat, N.; Gupta, S. V. J. Oncol. 2012, 84, 647206.

[3] Koeberle, A.; Northoff, H.; Werz, O. Biochem. Pharmacol. 2009, 77,1513

[4] Li, F.; Shanmugam, M. K.; Siveen, K. S. Oncotarget 2015, 6, 5147.

[5] Wu, A. T.; Lee, W. H.; Huang, C. C.; Lin, C. M.; Huang, Y. J.; Wei, L.; Ting, L. L.; Kuo, C. C.; Hsu, C.; Chiou, J. Biotechnol. Appl. Biochem. 2017, 64, 165.

[6] Liao, C. H.; Sang, S.; Liang, Y. C.; Ho, C. T.; Lin, J. K. Mol. Carcinog. 2004, 41, 140.

[7] Padhye, S.; Ahmad, A.; Oswal, N.; Sarkar, F. C. J. Hematol. Oncol. 2009, 2, 38 .

[8] Tanaka, T.; Kohno, H.; Shimada, R.; Kagami, S.; Yamaguchi, F.;
Kataoka, S.; Ariga, T.; Murakami, A.; Koshimizu, K.; Ohigashi, H. Carcinogenesis 2000, 21, 1183.

[9] Hong, J.; Sang, S.; Park, H. J.; Kwon, S. J.; Suh, N.; Huang, M. T.; Ho, C. T.; Yang, C. S. Carcinogenesis 2006, 27, 278.

[10] Ahmad, A.; Wang, Z.; Ali, R.; Maitah, M. Y.; Kong, D.; Banerjee, S.; Padhye, S.; Sarkar, F. H. J. Cell. Biochem. 2010, 109, 1134.

[11] Balasubramanyam, K.; Altaf, M.; Varier, R. A.; Swaminathan, V. Ravindran, A.; Sadhale, P. P.; Kundu, T. K. J. Biol Chem. 2004, $279,33716$.

[12] Ahmad, A.; Sarkar, S. H.; Aboukameel A.; Ali, S.; Biersack, B.; Seibt, S.; Li, Y. W.; Bao, B.; Kong, D.; Banerjee, S.; Schobert, R.; Padhye, S. B.; Sarkar, F. H. Carcinogenesis 2012, 33, 2450.

[13] Chen, X.; Zhang, X. Y.; Lu, Y.; Shim, J. Y.; Sang, S.; Sun, Z.; Chen, X. X. Nutr. Cancer. 2012, 64, 1211.

[14] Han, C. M.; Zhou, X. Y.; Cao, J.; Zhang, X. Y.; Chen, X. Bioorg. Chem. 2015, 60, 123.

[15] Socolsky, C.; Plietker, B. Chem.-Eur. J. 2015, 21, 3053.

[16] Biber, N.; Plietker, B.; Mows, K. Nat. Chem. 2011, 3, 938.

[17] Rodeschini, V.; Ahmad, N. M.; Simpkins, N. S. Org. Lett. 2006, 8, 5283.

[18] Nuhant, P.; David, M.; Pouplin, T.; Delpech, B.; Marazano, C. Org. Lett. 2007, 9, 287.

[19] Peng, P.; Linseis, L.; Winter, F. R.; Schmidt, R. R. J. Am. Chem. Soc. 2016, 138,6002 . 